\title{
OBSERVING SOLAR RADIO BURSTS FROM THE LUNAR SURFACE
}

\author{
R. J. MacDowall ${ }^{* \dagger}$, T. J. Lazio ${ }^{\ddagger \dagger}$, S. D. Bale ${ }^{\S \dagger}$, J. Burns ${ }^{\mathbb{I} \dagger}$, \\ N. Gopalswamy*, D. L. Jones ${ }^{\ddagger \dagger}$, M. L. Kaiser*, J. C. Kasper ${ }^{\| \dagger}$, and \\ K. W. Weiler**†
}

\begin{abstract}
Locating low frequency radio observatories on the lunar surface has a number of advantages, including fixed locations for the antennas and no terrestrial inteference on the far side of the moon. Here, we describe the Radio Observatory on the Lunar Surface for Solar studies (ROLSS), a concept for a near-side, low frequency, interferometric radio imaging array designed to study particle acceleration in the corona and inner heliosphere. ROLSS would be deployed during an early lunar sortie or by a robotic rover as part of an unmanned landing. The prime science mission is to image intense type II and type III solar radio bursts with the aim of determining the sites at and mechanisms by which the radiating particles are accelerated. Secondary science goals include constraining the density of the lunar ionosphere by searching for a low radio frequency cutoff of the solar radio emissions and detecting the low energy electron population in astrophysical sources. Furthermore, ROLSS serves as a pathfinder for larger, far-side lunar radio arrays, designed for faint sources.
\end{abstract}

\section{Introduction}

Key design requirements on ROLSS include the operational frequency and angular resolution. The electron densities in the solar corona and inner heliosphere are such that the relevant emission occurs below $10 \mathrm{MHz}$, essentially unobservable from Earth's surface due to the terrestrial ionospheric cutoff. Resolving the potential sites of particle acceleration requires an instrument with an angular resolution of at least $2^{\circ}$, equivalent to an array

\footnotetext{
* NASA Goddard Space Flight Center, Greenbelt, MD 20771, USA

$\dagger$ Lunar Science Institute, NASA/ARC, CA, USA

$\ddagger$ Jet Propulsion Laboratory, California Institute of Technology, Pasadena, CA 91109, USA

$\S$ Department of Physics, Univ. of California, Berkeley, CA 94720, USA

$\mathbb{I}$ Univ. of Colorado, Boulder, CO 80309, USA; Lunar Science Institute, NASA/ARC, CA, USA

\| Smithsonian Astrophysical Observatory, Harvard Univ., Cambridge, MA 02138, USA

**US Naval Research Laboratory, SW Washington, DC 20375, USA
} 
radius of approximately 500 meters. Operations would consist of data acquisition during the lunar day, with daily data downlinks. The major components of the ROLSS array are 3 antenna arms arranged in a Y shape, with a central electronics package (CEP). Each

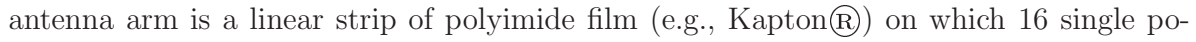
larization dipole antennas are located by depositing a conductor (e.g., silver). The arms also contain transmission lines for carrying the signals from the science antennas to the CEP. ROLSS is located on the lunar near-side, appropriate for observing intense solar radio bursts.

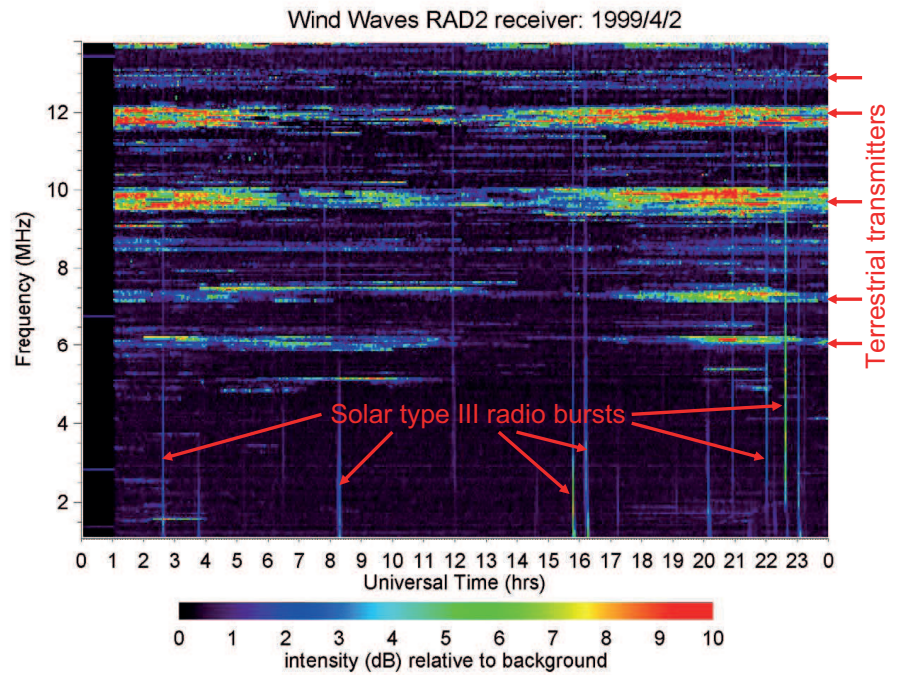

Figure 1: Observed low-frequency radio environment when the Wind spacecraft was near the moon. The 24-hr dynamic spectrum covers 1-14 MHz, with the intensity on a logarithmic color scale. The nearly vertical lines are type III radio bursts that are observed to continue to lower frequencies (not shown). Above $5 \mathrm{MHz}$, interference from ground-based transmitters is evident.

\section{Scientific Goals}

High-energy particle acceleration occurs in diverse astrophysical environments including the Sun and other stars, supernovae, black holes, and quasars. A fundamental problem is understanding the mechanisms and sites of this acceleration, in particular the roles of shock waves and magnetic reconnection. Within the inner heliosphere, solar flares and shocks driven by coronal mass ejections (CMEs) are efficient particle accelerators. 
Low frequency observations provide an excellent remote diagnostic because electrons accelerated in these regions can produce intense radio bursts. The intensities of these bursts make them easy to detect, as well as providing information about the acceleration regions. The radio burst mechanisms discussed here occur at the local plasma frequency $f_{p}=9 \sqrt{n_{e}}$ $\mathrm{kHz}$, or its harmonics, where $n_{e}$ is the electron density in $\mathrm{cm}^{-3}$. With a model for $n_{e}, f_{p}$ can be converted into a height above the corona, and changing $f_{p}$ can be converted into radial speed. Observations by widely-separated spacecraft have permitted triangulating the source locations.

Solar radio bursts are one of the primary remote signatures of electron acceleration in the inner heliosphere and our focus is on two emission processes, referred to as Type II and Type III radio bursts. Type II bursts originate from suprathermal electrons $(\mathrm{E}>100 \mathrm{eV})$ produced at shocks. These shocks are usually produced by CMEs as they expand into the heliosphere with Mach numbers greater than unity. Emission from a Type II burst drops slowly in frequency as the shock moves away from the Sun into lower density regions at speeds of $400-2000 \mathrm{~km} \mathrm{~s}^{-1}$. Type III bursts are generated by fast $(2-20 \mathrm{keV})$ electrons from magnetic reconnection, typically due to solar flares. As the fast electrons escape at a significant fraction of the speed of light into the heliosphere along open magnetic field lines, they produce emission that drops rapidly in frequency (see Figure 1).

Electron densities in the inner heliosphere yield relevant frequencies below $\sim 10 \mathrm{MHz}$. Observations must be conducted from space because the terrestrial ionosphere is opaque in this frequency range. Figure 1 illustrates the active low-frequency radio environment in space, including terrestrial radio frequency interference (RFI), as seen by the WAVES instrument on the Wind spacecraft [Bougeret et al., 1995]. Solar radio observations from the moon would necessarily take place in the gaps between the RFI.

Acceleration at Shocks: Observations of CMEs near Earth suggest electron acceleration generally occurs where the shock normal is perpendicular to the magnetic field [Bale et al., 1999], similar to acceleration at planetary bow shocks and other astrophysical sites. This geometry may be unusual in the corona, where the magnetic field is largely radial, as shown schematically in Figure 2a. There, the shock at the front of a CME generally has a quasi-parallel geometry $(\mathrm{Q}-\|)$. Acceleration along the flanks of the CME, where the magnetic field-shock normal is quasi-perpendicular $(\mathrm{Q}-\perp)$ would seem to be a more likely location for the electron acceleration and Type II emission. The radio array needs $\sim 2^{\circ}$ resolution to localize these acceleration site(s), yielding the preferred geometry (Q- $\|$ vs. Q- $\perp$ ) for radio emission around CMEs.

Electron and Ion Acceleration: Observations at 1-14 MHz made with the Wind spacecraft showed that complex Type III-L bursts are highly correlated with CMEs and intense (proton) solar energetic particle (SEP) events observed at 1 AU [Cane et al., 2002; Lara et al., 2003, MacDowall et al., 2003]. While an association between Type III-L bursts, proton SEP events, and CMEs is now confirmed, the electron acceleration mechanism remains poorly understood. Two competing sites for the acceleration have been suggested: at shocks in front of the CME or in reconnection regions behind the CME; see Figure 2b. For typical limb CMEs, the angular separation of the leading edge of the shock and the hypothesized reconnection region behind the CME is approximately $1.5^{\circ}$ when the CME shock is $3-4 \mathrm{R}_{\odot}$ from the Sun. 

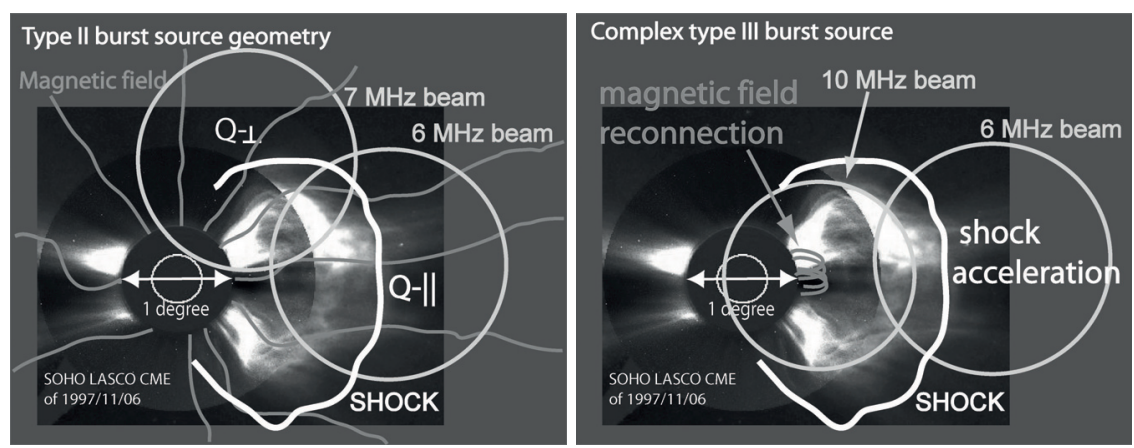

Figure 2: (a/Left) Where on the shock does electron acceleration occur, yielding type II radio emission? (b/Right) Are complex type III-L bursts produced by shock acceleration or reconnection behind the CME? Circles on the figures correspond to the radio beam width at the indicated frequencies for the $1 \mathrm{~km}$-diameter ROLSS array.

CME Interactions and Solar Energetic Particle (SEP) Intensity: Unusually intense radio emission can occur when successive CMEs leave the Sun within 24 hours, as if CME interaction produces enhanced particle acceleration [Gopalswamy et al., 2001, 2002]. Statistically associated with intense SEP events [Gopalswamy et al., 2004], this enhanced emission could result from more efficient acceleration due to changes in field topology, enhanced turbulence, or direct interaction of the CMEs. Lack of radio imaging makes it difficult to determine the nature of the interaction. Images with $\sim 2^{\circ}$ resolution would give Type II locations and permit identification of the causal mechanism and the relation to intense SEPs.

Lunar ionosphere The lunar ionosphere is a dynamic component of the tenuous lunar atmosphere, and one that can have a significant effect on plans to use low frequency imaging arrays to study solar and interplanetary radio sources. The existing data, mainly from dual-frequency radio occultation measurements, suggest that the density of free electrons is highly variable but can exceed $2000 \mathrm{~cm}^{3}$ (Figure 3). The implied local plasma frequency is about $0.4 \mathrm{MHz}$. The interpretation of these data is model dependent. Bauer [1996] concluded that the Luna data were consistent with no significant lunar ionosphere. However, ALSEP measurements during Apollo found a photoelectron layer near the surface with electron densities up to $10^{4} \mathrm{~cm}^{3}$ [Reasoner and O'Brien, 1972], implying a plasma frequency of 0.9 MHz. Such a high plasma frequency could affect radio imaging at frequencies up to a few $\mathrm{MHz}$.

Below the plasma frequency, radio waves cannot propagate. Consequently, as ROLSS tracks solar radio bursts to lower frequencies, it will also be able to search for a lunar ionosphere. If a low frequency cutoff (e.g., around a few $\mathrm{MHz}$ ) in the radio burst spectra were observed, this would be an indication of lunar ionospheric absorption and would place a constraint on the total electron column density of the lunar ionosphere.

Other goals: While not the prime mission of ROLSS, it may still be possible for ROLSS 


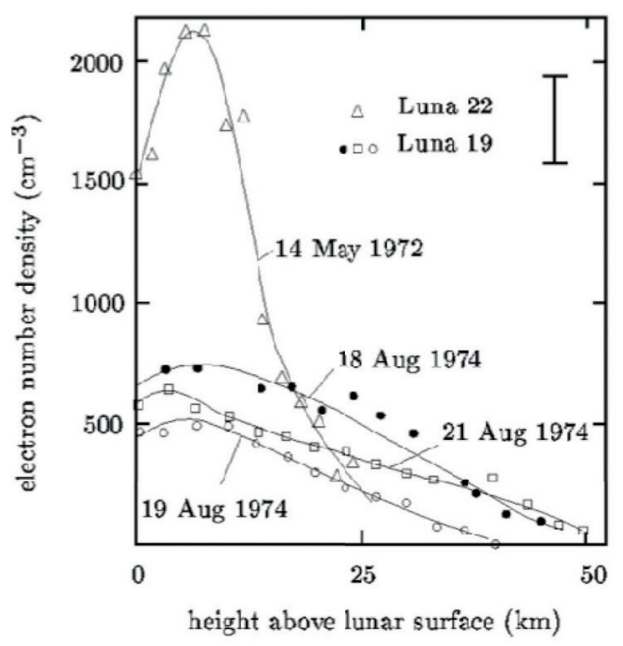

Figure 3: Lunar ionosphere electron densities derived from dual-frequency radio occultation measurements during the Luna 19 and Luna 22 missions [Vyshlov, 1976; Vyshlov and Savich, 1978].

to detect a few astronomical sources. The array should have a $(1 \sigma)$ sensitivity, in a 1hr integration at its higher frequencies, of about $10 \mathrm{Jy}$. By contrast, the stronger radio astronomical sources (e.g., 3C sources) should have flux densities approaching $500 \mathrm{Jy}$, with the very strongest having flux densities exceeding several thousand Janskys.

For cosmological studies resolving structures in the early universe a much larger array with thousands of antennas is required. ROLSS would serve as a pathfinder for such larger arrays.

\section{Implementation}

Overview of the ROLSS concept: The array consists of 3 arms arranged in a Y configuration, subject to local topographic constraints. Each arm is $500 \mathrm{~m}$ long, providing $\sim 2^{\circ}$ angular resolution at $30-\mathrm{m}$ wavelength $(10 \mathrm{MHz})$. The arms themselves consist of a polyimide film (PF) on which electrically-short dipole antennas are deposited, and they hold the transmission system for sending the electrical signals back to the central electronics package (CEP), located at the intersection of the arms. The CEP performs the requisite filtering and digitization of the signals, then downlinks them to Earth for final imaging and scientific analysis.

The array operates over the wavelength range 30-300 $\mathrm{m}(1-10 \mathrm{MHz})$, with a selectable, variable frequency sub-band that can be placed anywhere within the operational wave- 
length range. During the course of the concept study, the NASA/GSFC Instrument Design Laboratory (IDL) provided an intensive engineering study of the ROLSS concept. We refer to the conclusions of that study as output from the "IDL run."
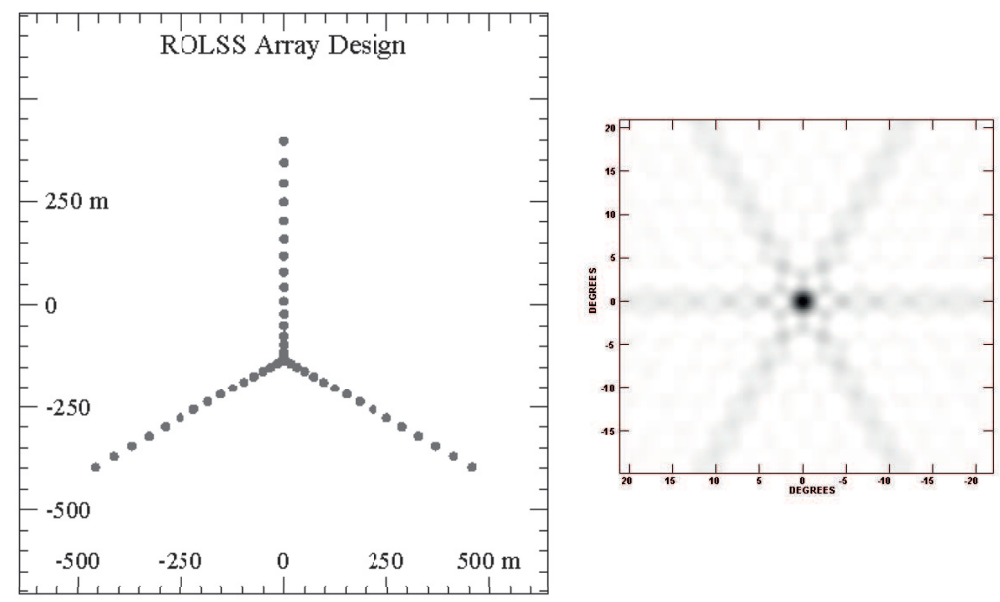

Figure 4: (a/Left) Nominal science antenna distribution along the antenna arms. (b/Right) The resulting point-spread function ("beam") for a snapshot image. The maximum sidelobe is at 5.9 $\mathrm{dB}$ and the rms sidelobe level is $15 \mathrm{~dB}$, sufficient to achieve ROLSS goals.

Figure 4a shows a nominal layout of 16 science antennas along each arm. Standard radio astronomical software within the Astronomical Image Processing Software (AIPS) package of the National Radio Astronomy Observatory (NRAO) was used to simulate the instantaneous or "snapshot" point spread function (PSF) or "beam" of the ROLSS array, assuming 16 antennas on each arm and logarithmic spacing along the arm. The 6-arm "star" pattern of the beam in Figure 4b reflects the Y shape of the ROLSS array. The dynamic range in the beam - defined as the ratio between the peak and the rms level - is $15 \mathrm{~dB}$. This dynamic range is consistent with a simple estimate that the rms level in an interferometric image should be of order $1 / N$ if the array consists of $\mathrm{N}$ antennas. For $\mathrm{N}$ $\sim 50(\sim 16 \times 3)$, the expected rms level is $2 \%(-17 \mathrm{~dB})$ of the peak.

Antenna and substrate design: The ROLSS science antennas consist of single polarization, electrically short dipoles, deposited on a PF. The transmission leads to the CEP are also deposited on the PF. The PF is flexible enough to be stored in a roll during transit and deployed directly on the lunar surface by unrolling.

The lunar regolith has a typical relative dielectric permittivity of $2-3$ (for lunar soils), high resistivity, and a large skin depth at low radio frequencies. Consequently, it is possible for a low frequency radio antenna to operate when placed directly on the lunar surface. Modeling suggest using a nominal length of 14 meters for the ROLSS dipoles. The antenna resonant frequency will be near the high end of the ROLSS observing frequency range. 
At the low end of this range, the antenna will be electrically short and will have a small feed point resistance and high capacitive reactance. The radiation resistance, efficiency, and fractional bandwidth will all be very small, thereby reducing the sensitivity, but the ROLSS science goals can still be met. The baseline design has 16 antennas per arm spaced logarithmically in order to provide a range of Fourier spacings for good imaging. The nominal topology is a wide dipole, with $\sim 1.5$ - $\mathrm{m}$ width, which helps provide a broad wavelength response.

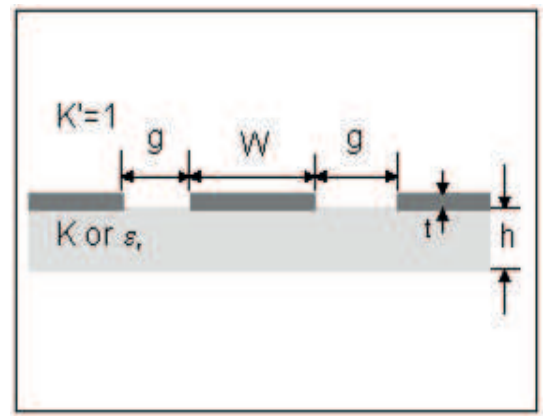

Figure 5: Cross section of the transmission line. Light gray indicates the substrate; dark gray, the deposited metal. (Figure and values from IDL run: $w=$ width of center conductor $=1.44$ $\mathrm{mm}, g=g a p=1.3 \mathrm{~mm}, t=C u$ thickness $=0.010 \mathrm{~mm}$; the Ks indicate dielectric constants, $h$ is the substrate thickness $=0.025 \mathrm{~mm}$ ).

Signal transmission to the central hub: Each of the dipoles is connected to the CEP at the end of the PF by transmission lines deposited on the film. Each transmission line is a coplanar waveguide structure ( $\sim 6 \mathrm{~mm}$ wide), see Figure 5 . Simulations, conducted as part of the IDL run, indicate that a gap between the lines reduces cross coupling and suggest that the lines should be placed symmetrically to reduce antenna pattern distortions. These simulations suggest $\sim 25 \mathrm{~dB}$ of signal loss will occur on the longest transmission lines at $10 \mathrm{MHz}$, which must be compensated for at the receiver. Investigation of using active elements on the PF and alternate signal transmission line designs is ongoing.

The width of the transmission lines is determined in part by the requirement of mitigating micrometeorite damage. During the IDL run, estimates of the micrometeorite damage were determined by scaling from experience with the Hubble Space Telescope (HST) Wide Field/Planetary Camera $(\mathrm{WF} / \mathrm{PC}$ ) radiator. The estimated bombardment rate at the Moon's surface is $4.9 \mathrm{~m}^{-2} \mathrm{yr}^{-1}$ for impacts producing craters up to $1 \mathrm{~mm}$ wide. Larger craters are sufficiently rare that none were seen in a 3.6-yr exposure. The longest transmission lines are likely to receive a few impacts per year $(\sim 3)$, but none are likely to be so large that the transmission line will be completely severed.

Preamplifiers, receivers, data processing, and data storage: The ROLSS CEP (shown in Figure 6) houses all of the post science data transmission functions including amplification, data acquisition hardware, power supply electronics, telemetry communications system, and the thermal management system for these components. When deployed, 
the CEP stands $\sim 1 \mathrm{~m}$ above the lunar surface so that the thermal management system is not subject to dust contamination. The swept-frequency receiver system will be frequency agile and record $\sim 10 \log$-spaced frequencies at 2-bit resolution and typically $2 \%$ bandwidth (of central frequency), providing the incident wave phase at each antenna (20 $\mathrm{MHz}$ sampling at $10 \mathrm{MHz}$ ). We anticipate $15 \mathrm{sec}$ of sampling per frequency, which may require multiple receivers to cover the type III burst event with adequate time resolution.

Data downlink: ROLSS uplink telemetry and commanding would be handled by an Sband uplink. A nominal configuration has a 9-m diameter or larger antenna at a site such as GSFC Wallops. The ROLSS CEP would contain an S-band transceiver, which could also be used for data downlink in emergency situations. Data downlink would typically be handled by a Ka-band system. The ROLSS CEP would host a 0.6-m diameter parabolic gimbaled antenna. The conclusion of the IDL run was that a Ka-band downlink via such an antenna provided the current optimum based on downlink data requirements, weather (rain) conditions, and likely ground-station antenna availability.

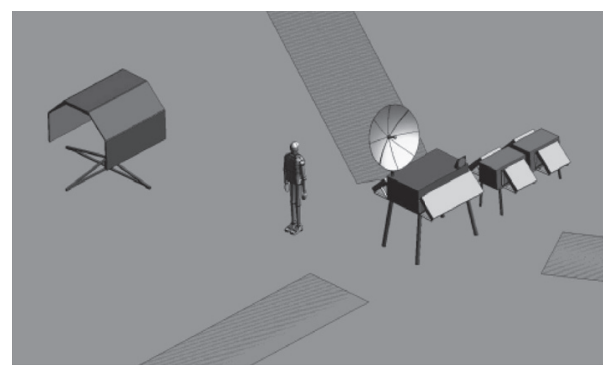

Figure 6: ROLSS Central Electronics Package (CEP) layout showing relative sizes of electronics boxes with thermal louvers, high-gain antenna, and solar arrays (left). (Figure from IDL run.)

Siting and deployment: The prime science mission for ROLSS is solar observations, which favors a near-side, equatorial site. A polar site would require that one or more arms of ROLSS be longer than the nominal $500 \mathrm{~m}$ length in order to compensate for the foreshortening of the array and maintain the same angular resolution. The site itself should be of relatively low relief topography (e.g., the surface of a mare vs. the highlands). We emphasize that the entire site does not have to be flat as the fraction of the area occupied by the antenna arms is relatively small - for a circular area with a radius of 500 $\mathrm{m}$, the antenna arms occupy only $0.3 \%$ of the total area. Further, there is no requirement on the absolute orientation of the arms. Rotating the arms about the center of the array merely rotates the beam pattern on the sky. The relative orientation of the arms is required to be $120^{\circ} \pm 3^{\circ}$, with the error determined by requiring that the nominal antenna position not vary by more than 1 wavelength at the extreme end of an arm. This level of alignment was demonstrated during the Apollo missions. Only modest requirements are set on the smoothness of the antenna arm locations. The shortest operational wavelength for ROLSS would be $\sim 30 \mathrm{~m}$, and an individual science antenna would be $15 \mathrm{~m}$ in length. Requiring that the antenna shapes are not distorted by more than $\lambda / 10$ implies that deployment requires linear extents of $\sim 500 \mathrm{~m}$ with elevation variations not larger than 
$\sim 3$ meters along the intended antenna arm positions. We assume that on the timescale of likely deployment for ROLSS any site will have better than meter-scale resolution images available (post-Lunar Reconnaissance Orbiter).

The ROLSS deployment itself could be done completely robotically, by astronauts on a crewed rover, or with a mix of these two modes. A completely robotic deployment would allow ROLSS to be deployed prior to any human sortie missions. Scaling from existing rovers, a ROLSS rover could easily be designed to carry and deploy tens of kilograms of antenna mass.

Other ROLSS concept elements: The ROLSS mission concept calls for lunar day operations only; there are no nighttime operations. The baseline ROLSS design assumes a solar panel assembly for power generation. Multiple load profiles were generated during the IDL run, with the final load profile suggesting that an average $125 \mathrm{~W}$ load (peak 167 W load) would need to be supported by solar panels.

Thermal management is a key requirement for lunar-based instrumentation. The antenna arms are entirely passive and require no thermal management. The CEP is equipped with a set of radiators in order to maintain the internal electronics at a temperature below their assumed maximum operating temperature $\left(80^{\circ} \mathrm{C}\right)$. These radiators are equipped with thermal louvers, outfitted with actuators that sense radiator temperature and rotate thin aluminum blades to open or closed positions. When open, the louvers allow the radiators to dissipate heat. During lunar night, batteries provide survival heating.

Other radio imaging options: In this paper, we have focused on the polyimide film antenna system, but other viable approaches for antennas on the lunar surface are also being studied. Also, a constellation of microsats carrying dipole antennas is a possible alternative for low-frequency radio imaging [MacDowall et al., 2006].

\section{Conclusion}

Investigation of particle acceleration and propagation using radio bursts as diagnostic tools has been performed to date without imaging capability below $\sim 20 \mathrm{MHz}$. A radio observatory such as ROLSS, located on the near-side lunar surface, would provide the first images of radio bursts in the outer solar corona and interplanetary space, yielding new information on solar flaring and shock acceleration of particles. ROLSS observations would also measure the lunar ionosphere electron density, detect the brightest galaxies, and demonstrate the capability of lunar-based radio imaging for larger radio arrays.

\section{References}

Bale, S.D., M. J. Reiner, J.-L. Bougeret, M. L. Kaiser, S. Krucker, D. E. Larson, and R. P. Lin, The Source Region of an Interplanetary Type II Radio Burst, Geophys. Res. Lett., 26, 1573, 1999. 
Bauer, S. J., Limits to a Lunar Ionosphere, Sitzungsberichte und Anzeiger, Abt. 2, 133, 17, 1996.

Bougeret, J.-L., M. L. Kaiser, P. J. Kellogg, R. Manning, K. Goetz, S. J. Monson, N. Monge, L. Friel, C. A. Meetre, C. Perche, L. Sitruk, and S. Hoang, Waves: The Radio and Plasma Wave Investigation on the Wind Spacecraft, Space Sci. Rev., 71, 231, 1995.

Cane, H. V., W. C. Erickson, and N. P. Prestage, Solar Flares, Type III Radio Bursts, Coronal Mass Ejections, and Energetic Particles, J. Geophys. Res., 107, 1315, 2002.

Gopalswamy, N., S. Yashiro, M. L. Kaiser, R. A. Howard, and J.-L. Bougeret, Radio Signatures of Coronal Mass Ejection Interaction: Coronal Mass Ejection Cannibalism?, Astrophys. J., 548, L91, 2001.

Gopalswamy, N., S. Yashiro, M. L. Kaiser, R. A. Howard, and J.-L. Bougeret, Interplanetary Radio Emission due to Interaction Between Two Coronal Mass Ejections, Geophys. Res. Lett., 29, 080000-1, 2002.

Gopalswamy, N., S. Yashiro, S. Krucker, G. Stenborg, and R. A. Howard, Intensity Variation of Large Solar Energetic Particle Events Associated with Coronal Mass Ejections, J. Geophys. Res. (Space Physics), 109, 12105, 2004.

Lara, A., N. Gopalswamy, S. Nunes, G. Muñoz, and S. Yashiro, A Statistical Study of CMEs Associated with Metric Type II Bursts, Geophys. Res. Lett., 30, 120000-1, 2003.

MacDowall, R. J., A. Lara, P. K. Manoharan, N. V. Nitta, A. M. Rosas, and J. L. Bougeret, Long-duration hectometric type III radio bursts and their association with solar energetic particle (SEP) events, Geophys. Res. Lett., 30, 12, 8018, doi:10.1029/ 2002GL016624, 2003.

MacDowall, R. J., N. Gopalswamy, M.L. Kaiser, L.D. Demaio, S. D. Bale, J. Hewitt, J. C. Kasper, A. J. Lazarus, R. E. Howard, D. L. Jones, M. J. Reiner, and K. W. Weiler, Microsat and lunar-based imaging of solar radio bursts, in Planetary Radio Emissions VI, edited by H. O. Rucker, W. S. Kurth, and G. Mann, Austrian Academy of Sciences Press, Vienna, 491-504, 2006.

Reasoner, D. L., and B. J. O'Brien, Measurement on the Lunar Surface of Impact-Produced Plasma Clouds, J. Geophys. Res., 77, 1292, 1972.

Vyshlov, A.S., Preliminary Results of Circumlunar Plasma Research by the Luna 22 Spacecraft, Space Research XVI, Proc. Open Meetings of Workshop Groups of Physical Sciences, Akademie-Verlag, 945, 1976.

Vyshlov, A. S., and N. A. Savich, Observations of Radio Source Occultations by the Moon and the Nature of the Plasma near the Moon, Cosm. Res., 16, 551, 1978. 